\title{
A Guided Tour in Vibration Control: From Large Civil Structures to Precision Mechatronics
}

\author{
A. Preumont \\ Université Libre de Bruxelles (ULB) \\ Active Structures Laboratory, \\ Brussels, Belgium
}

The first part of the lecture addresses various problems related to the vibration of large civil engineering structures. The case of suspension bridges is of special interest, because they are complex nonlinear structures and they are particularly sensitive to vibrations.

The second part of the lecture considers various problems of precision mechatronics associated with space telescopes: (i) the vibration isolation of precision payloads (disturbances introduced by the attitude control and cryo-coolers are responsible for jitter of the line of sight), and (ii) Adaptive Optics for wavefront correction.

The final part of the lecture focuses on the control-structure interaction of future extremely large telescopes; these new specific problems result from the extreme precision (requiring a large control bandwidth) and very large size of the structures (increasing flexibility). 